

\title{
Lower-limb performance disparities: Implications for exercise prescription in multiple sclerosis
}

\author{
Rebecca D. Larson, PhD; ${ }^{*}$ Kevin K. McCully, PhD; Daniel J. Larson, PhD; William M. Pryor, MS; Lesley J. \\ White, $\mathbf{P h D}$ \\ Department of Kinesiology, University of Georgia, Athens, GA
}

\begin{abstract}
The purpose of this study was to examine unilateral lower-limb exercise tolerance during fixed-load cycling to quantify performance disparities of the legs. Eight individuals with relapsing-remitting multiple sclerosis (MS) and seven controls performed submaximal single-leg cycling. Individuals with MS performed significantly more work with the stronger leg than the weaker leg (stronger leg: $6.4+/-1.7 \mathrm{~kJ}$, weaker leg: $4.7+/-2.5 \mathrm{~kJ}, p=0.02)$. The control group displayed no statistical differences between limbs $(p=0.36)$. These results highlight a need for individualized exercise testing when prescribing training programs for those with MS.
\end{abstract}

Key words: bilateral, exercise prescription, leg performance, leg performance disparities, lower limb, lower-limb exercise, MS, multiple sclerosis, muscle, work.

\section{INTRODUCTION}

Individuals with multiple sclerosis (MS) exhibit higher relative oxygen consumption and heart rates than matched controls at the same walking speeds [1] and incremental exercise workloads [2], suggesting greater metabolic stress [1-2]. Evidence suggests that individuals with MS exhibit bilateral differences in strength and function [3-5], which may help explain, in part, higher energy demand during exercise that could contribute to early onset muscle fatigue and reduced exercise tolerance. A 2005 case study by White and Dressendorfer reported bilateral differences in leg strength and maximal oxygen uptake in an individual with MS who exhibited left leg exercise-induced monoparesis [4]. Chung et al. in 2008 observed that lower knee extensor power asymmetry was greater in individuals with MS than in controls [3]. Unilateral leg weakness has also been observed in individuals with MS [6-7]. In a 2011 study, Larson and White observed bilateral differences in hip bone density in ambulatory individuals with MS, which may illustrate consequences of altered bilateral function [8]. Additionally, Larson et al. in 2013 observed that individuals with MS exhibited significant leg asymmetry in strength, oxidative capacity, and work performed, suggesting that the magnitude of bilateral differences might limit functional abilities [5].

Further research related to potential bilateral differences in limb performance is needed because it might improve our understanding of functional limitations for people with MS. This new information could advance the development of effective prevention and rehabilitation

\footnotetext{
Abbreviations: $\mathrm{CI}=$ confidence interval, EDSS $=$ Expanded Disability Status Scale, MFIS = Modified Fatigue Impact Scale, MS = multiple sclerosis, MVIC = maximal voluntary isometric contraction, POMS-B = Profile of Mood States Brief Version, RRMS = relapsing-remitting MS, SD = standard deviation, $\mathrm{VO}_{2}$ peak = peak oxygen uptake.

*Address all correspondence to Rebecca D. Larson, PhD; Department of Health and Exercise Science, University of Oklahoma, Norman, OK 73019; 405-325-6325; fax: 405325-0594. Email: rdlarson@ou.edu
}

http://dx.doi.org/10.1682/JRRD.2013.09.0191 
strategies to attenuate fatigue and premature disability. Such information warrants consideration when designing exercise programs for people with MS wherein intensity and duration may be outcomes. Differences in lowerlimb function can result in limb-loading inequities when conventional exercise program guidelines are used to determine exercise intensity.

The purpose of this study was to examine unilateral lower-limb exercise tolerance during fixed-load cycling to quantify potential performance disparities of the legs. We hypothesized that individuals with MS would exhibit performance disparities between legs.

\section{METHODS}

\section{Participants}

Fifteen volunteers participated: eight with MS (6 women and 2 men) and seven age- and sex-matched, non-MS controls ( 5 women and 2 men). Participants with MS were included if they had a clear diagnosis of relapsing-remitting MS (RRMS) and an Expanded Disability Status Scale (EDSS) score of $<6.5$ (ambulatory without aid) [9]. Each participant had physician's clearance and signed a consent form approved by the University of Georgia Institutional Review Board prior to participation.

\section{Experimental Design}

This investigation was part of a larger study that involved physical performance testing [5]. All participants had a defined stronger leg and weaker leg based on leg extensor strength assessments. This study involved a total of five testing visits (visit 1: screening and familiarization with strength and single-leg cycling, visit 2: leg strength assessment and additional single-leg cycling practice, visit 3: whole body oxygen uptake cycling test, and visits 4 and 5: submaximal fixed single-leg cycling). The testing order of the legs (stronger/weaker) was randomized. Participants were tested at approximately the same time of day and were asked to abstain from alcohol, caffeine, smoking, and exercise $12 \mathrm{~h}$ before their testing visits, which were separated by a minimum of $48 \mathrm{~h}$ to ensure adequate recovery. Additionally, because the legs were tested on different days, daily screening of fatigue was performed using questionnaires related to fatigue (Modified Fatigue Impact Scale [MFIS] Physical Domain) and the Profile of Mood States Brief Version (POMS-B). If scores were 2.5 standard deviations (SDs) higher than the participant's previous scores on either the MFIS Physical Domain or the POMS-B, he or she was retested at a later date once fatigue levels returned to normal levels. Physical activity was measured using a questionnaire that asked about frequency, duration, and intensity of physical activity during a typical week. Whole body and limb-specific lean and fat mass were measured by dual-energy X-ray absorptiometry (iDXA, GE Healthcare-Lunar; Madison, Wisconsin).

\section{Muscle Strength}

Maximal voluntary isometric contractions (MVICs) of the quadriceps of each leg were assessed using a custom chair that set the knee angle at a constant $70^{\circ}$; the thigh and torso were stabilized during testing. The chair's lever arm was connected to a force transducer, and the digitized signal was recorded using MATLAB (MathWorks; Natick, Massachusetts). Three submaximal contractions followed by a 5 min rest period were performed before three MVICs. The highest MVIC value was used to determine which was the strong/less affected leg and which was the weak/more affected.

\section{Whole Body Oxygen Uptake}

Conventional incremental cycle ergometer (Lode; Groningen, the Netherlands) test measured peak oxygen uptake $\left(\mathrm{VO}_{2}\right.$ peak) under climate-controlled conditions. After a standard warm-up and 5 min rest period, participants cycled at $25 \mathrm{~W}$, increasing $15 \mathrm{~W}$ every minute until one of our defined test termination criteria was met: (1) any symptom impairing ability to continue, (2) volitional exhaustion, or (3) pedaling rate below $40 \mathrm{rpm}$. Expired gases were measured continuously using a calibrated metabolic cart (TrueMax 2400, Parvo Medics; Salt Lake City, Utah). Heart rate was recorded by telemetry (Polar RXS 800, Polar Electro Inc; Lake Success, New York) at the end of each stage. Peak workload was defined as the highest workload sustained for at least $30 \mathrm{~s}$.

\section{Submaximal Fixed-Load Cycling}

Single-leg performance was assessed using a submaximal fixed-load exercise test with prior familiarization. The order of right and left leg testing was randomized. Following the same warm-up, participants performed a single-leg 5 min cycling bout of exercise at 20 percent of peak whole body peak workload as determined from previous data [4]. During this test, the foot 
was securely fastened to the pedal and the nonexercising leg was positioned on a chair to ensure nonparticipation.

\section{Statistical Analyses}

The data were initially screened using frequency distributions and estimates of skewness and kurtosis to verify that our data were normally distributed. Analyses were performed using SPSS software version 17.0 (IBM Corporation; Armonk, New York). Independent $t$-tests were used to detect differences across group differences. Dependent $t$-tests were used to compare across-limb differences for total work performed. Data are expressed as mean \pm SD. An alpha of 0.05 was used for statistically significant differences. Confidence intervals (CIs) were reported and represent either the $\mathrm{CI}$ for the mean difference in groups (MS - controls) or CI for the mean difference in legs (stronger leg - weaker leg).

\section{RESULTS}

\section{Participant Characteristics}

Eight individuals with MS (6 women and 2 men) and seven healthy controls (5 women and 2 men) completed the study. The mean EDSS score was $2.6 \pm 1.6$, ranging from 0 to 5 (one participant was a 0 , one was a 1 , one was a 2 , three participants were 3 s, one was a 4 , and one was a 5), indicating mild to moderate impairment [9]. Disease duration was $12.6 \pm 8.1 \mathrm{yr}$ (ranging from 6 to $31 \mathrm{yr}$ ). Anthropometric data are shown in Table 1. No statistically significant differences existed between the groups $(p>0.05)$ (Table 1).

\section{Whole Body Oxygen Uptake}

Groups were not different for $\mathrm{VO}_{2}$ peak (milliliters per kilogram per minute), peak workload (watts), respiratory exchange ratio, peak lactate, and ventilation (liters per minute) $(p>0.05)$. However, the individuals with MS achieved a significantly lower maximal heart rate (beats per minute) than the controls during the exercise test ( $p=$ 0.02 ), with a Cohen $d$ effect size of -1.36 (which is considered large [10]). Despite not reaching statistical significance between groups, $\mathrm{VO}_{2}$ peak and peak workload had large effect sizes ( -0.98 and -0.97 , respectively) (Table 2$)$.

\section{Leg Characteristics: Composition and Strength}

Leg lean and fat mass were not statistically different between legs or groups $(p>0.05)$. The mean between-leg

Table 1.

Characteristics of subjects with multiple sclerosis (MS) and controls.

\begin{tabular}{lccccl}
\hline Variable & MS $(\boldsymbol{n}=\mathbf{8})$ & Control $(\boldsymbol{n}=\mathbf{7})$ & $\boldsymbol{p}$-Value & Group Difference & CI \\
\hline Age $(\mathrm{yr})$ & $51.1 \pm 9.2$ & $49.4 \pm 14.3$ & 0.74 & 2.3 & $(-10.9,15.5)$ \\
Height $(\mathrm{cm})$ & $167.5 \pm 7.5$ & $169.1 \pm 9.0$ & 0.72 & -1.5 & $(-10.7,7.6)$ \\
Body Mass $(\mathrm{kg})$ & $70.4 \pm 13.8$ & $75.4 \pm 30.4$ & 0.74 & -4.1 & $(-29.9,21.7)$ \\
Body Mass Index $\left(\mathrm{kg} / \mathrm{m}^{2}\right)$ & $25.0 \pm 3.9$ & $26.0 \pm 8.3$ & 0.77 & -1.0 & $(-8.1,6.1)$ \\
Fat Mass (\%) & $39.4 \pm 6.1$ & $36.9 \pm 8.7$ & 0.52 & 2.6 & $(-5.7,10.8)$ \\
\hline
\end{tabular}

Note: Data are mean \pm standard deviation. Group difference = mean of MS - mean of control. Confidence interval (CI) represents differences in group means.

Table 2.

Metabolic and associated measures during whole body cycling test for subjects with multiple sclerosis (MS) and controls.

\begin{tabular}{|c|c|c|c|c|c|}
\hline Variable & MS $(n=8)$ & Control $(n=7)$ & $p$-Value & $\begin{array}{c}\text { Group } \\
\text { Difference }\end{array}$ & CI \\
\hline $\mathrm{VO}_{2}$ peak (mL/kg/min) & $19.3 \pm 4.9$ & $26.1 \pm 8.4$ & 0.07 & -6.8 & $(-14.3,0.7)$ \\
\hline Peak Workload (W) & $115.0 \pm 28.9$ & $155.0 \pm 51.8$ & 0.10 & -40.7 & $(-86.6,5.2)$ \\
\hline Peak RER $\left(\mathrm{VCO}_{2} / \mathrm{VO}_{2}\right)$ & $1.18 \pm 0.17$ & $1.17 \pm 0.1$ & 0.96 & 0.004 & $(-0.2,0.2)$ \\
\hline Peak Lactate (mmol/L) & $5.7 \pm 2.5$ & $6.7 \pm 1.8$ & 0.38 & -1.0 & $(-3.5,1.4)$ \\
\hline HR Max (bpm) & $138.0 \pm 15.7$ & $162.1 \pm 19.3$ & $0.02^{*}$ & -21.2 & $(-42.1,-0.3)$ \\
\hline
\end{tabular}

Note: Data are mean \pm standard deviation. Group difference = mean of MS - mean of control. Confidence interval (CI) represents differences in group means.

*Statistically significant differences in group means at $p<0.05$.

$\mathrm{HR} \max =$ maximal heart rate, $\mathrm{RER}=$ respiratory exchange ratio, $\mathrm{VCO}_{2}=$ carbon dioxide uptake, $\mathrm{VE}=$ ventilation, $\mathrm{VO}_{2}=$ oxygen uptake . 
difference in strength (stronger leg - weaker leg) was not statistically different between groups (MS: $18.3 \pm 12.5$, control: $4.6 \pm 13.3, p>0.05$; Table 3). (Figure 1 displays the individual data for the MS group.) Additionally, the median difference for the MS group was $16.6 \mathrm{lb}$ compared with $4.8 \mathrm{lb}$ in the control group.

\section{Submaximal Fixed-Load Cycling}

Individuals with MS performed significantly more work with the stronger leg during the submaximal $5 \mathrm{~min}$ test (stronger leg: $6.4 \pm 1.7 \mathrm{~kJ}$, weaker leg: $4.7 \pm 2.5 \mathrm{~kJ}, p=$ 0.02 , Cohen $d$ effect size $=0.99$, which is considered large [9]). (Figures 2-3 display the individual data for the MS group.) The control group displayed no statistical between-leg differences in their ability to complete the fixed-load cycling trial (stronger leg: $9.2 \pm 3.2 \mathrm{~kJ}$, weaker leg $9.1 \pm 3.2 \mathrm{~kJ}, p=0.36$, Cohen $d$ effect size $=-0.13$, which is considered small [10]; Table 4). The betweenleg difference in work performed (stronger leg - weaker leg) was statistically different between groups (MS: $1.69 \pm$ $1.60 \mathrm{~kJ}$, control: $0 \pm 0.40, p=0.03$ ). (Figure 3 displays the individual data for the MS group.) Additionally, the median between-leg difference for the MS group was $1.8 \mathrm{~kJ}$ compared with $0 \mathrm{~kJ}$ in the control group.

\section{DISCUSSION AND STUDY LIMITATIONS}

In our study of ambulatory people with MS, the major finding was that work during a 5 min exercise test was significantly different between legs in people with MS, but not in control participants. People with MS often experience decrements in motor drive, which often affects the lower limbs disproportionally [11]. Activities of daily living such as walking require sufficient syn- chronization of bilateral motor unit recruitment and discharge rates. Typically, the legs are recruited bilaterally and limb preference may switch depending on the complexity and conditions during the movement [12]. Individuals with MS can be limited in their ability to activate motor units during bilateral movements, which may result in the development of an imbalance in function and performance. The inability to bilaterally modulate and produce motor discharge rates appropriately during exercise could result in further interlimb differences contributing to reductions in exercise capacity and increased levels of premature muscle failure. Our data support this, as evidenced by the inability of the weaker leg to perform the same work as the stronger leg.

The inability of the weaker leg in the MS group to complete the 5 min submaximal fixed-load cycling test may be further evidence of a compensatory motor recruitment switching to the stronger/less affected leg. Comparing workloads during the fixed-load ride to the unilateral incremental test, we found that the weaker leg was actually exercising at approximately 49 percent (range: 24\%-104\%) of maximal workloads, while the group's stronger leg was exercising at only 32 percent (range: 25\%-58\%). In comparison, the control group was exercising at approximately the same relative intensity for both legs (32\%). The inability of the weaker leg to maintain the effort indicates a premature muscle fatigue/ task failure not observed in the stronger leg or in either leg of the control group. The premature failure observed in the weaker leg of the MS group may, in part, be explained because it was exercising at a higher relative intensity as a result of altered neurological function. However, our study was not able to separate central and peripheral influences on the observation of premature failure in the weaker leg. A combination of these factors

Table 3.

Differences between limbs for subjects with multiple sclerosis (MS) and controls.

\begin{tabular}{lccccc}
\hline Variable & $\begin{array}{c}\text { MS Legs } \\
(\mathbf{S}-\mathbf{W})(\boldsymbol{n}=\mathbf{8})\end{array}$ & $\begin{array}{c}\text { Control Legs } \\
(\mathbf{S}-\mathbf{W})(\boldsymbol{n}=\mathbf{7})\end{array}$ & $\boldsymbol{p}$-Value & $\begin{array}{c}\text { Group } \\
\text { Difference }\end{array}$ \\
\hline Lean Mass (kg) & $0.2 \pm 0.41$ & $-0.1 \pm 0.3$ & 0.11 & 0.3 & $(-0.01,0.7)$ \\
Fat Mass (kg) & $0.05 \pm 0.2$ & $0.3 \pm 0.3$ & 0.81 & -0.2 & $(-0.2,0.2)$ \\
MVIC (lb) & $18.3 \pm 12.5$ & $4.6 \pm 13.3$ & 0.06 & 13.8 & $(-0.7,28.2)$ \\
Work (kJ) & $1.7 \pm 1.6$ & $0.1 \pm 0.4$ & $0.02^{*}$ & 1.5 & $(0.4,2.9)$ \\
\hline
\end{tabular}

Note: Data are mean \pm standard deviation. $\mathrm{S}-\mathrm{W}=$ difference between stronger $(\mathrm{S})$ and weaker $(\mathrm{W})$ legs. Group difference = mean difference in groups (mean difference of MS legs - mean difference of control legs). Confidence interval (CI) = differences between groups. Lean and fat mass from dual-energy X-ray absorptiometry. Work = total work performed during 5 min submaximal single-leg cycling.

* Statistically significant differences between groups at $p<0.05$.

MVIC = maximal voluntary isometric contraction. 


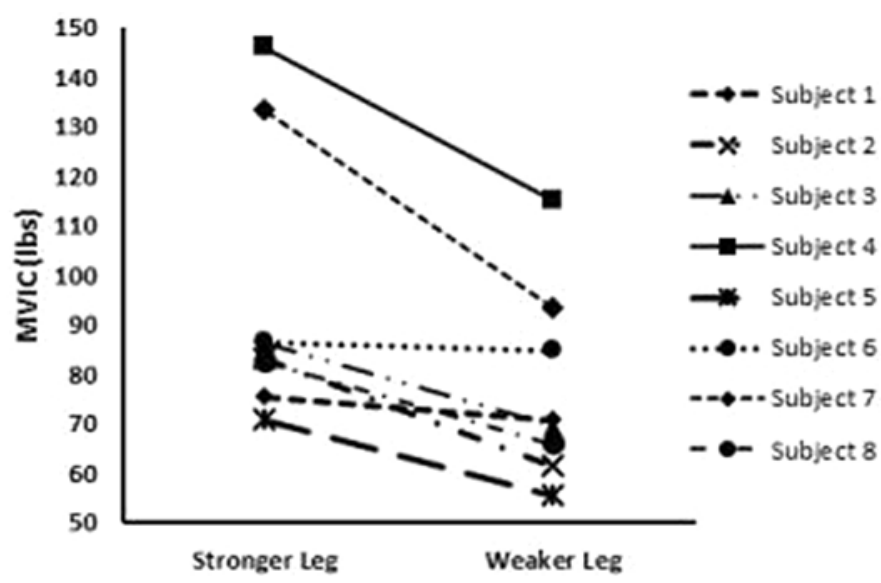

Figure 1.

Maximal voluntary isometric contraction (MVIC) strength for stronger and weaker legs for 8 participants with multiple sclerosis.

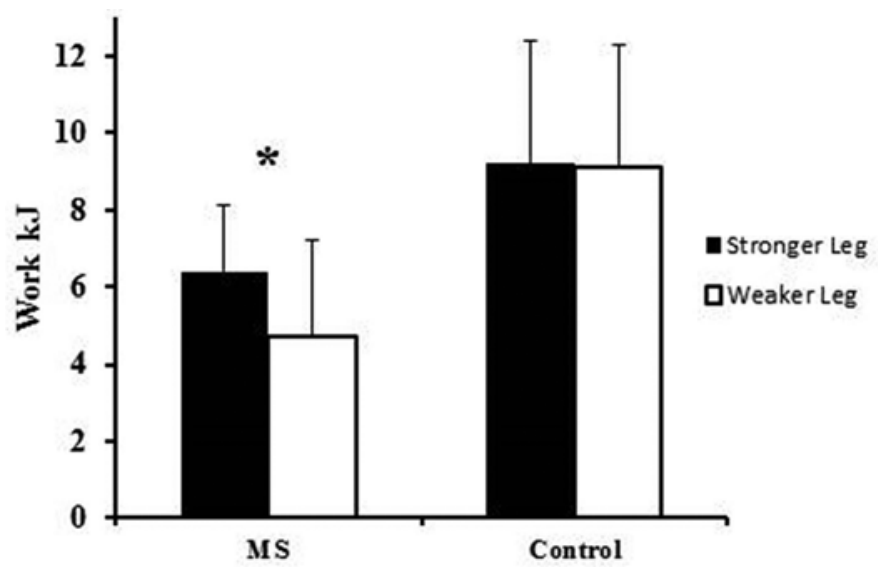

Figure 2.

Total work performed during single-leg 5 min cycling test in kilojoules. Values are mean \pm standard deviation. MS $=$ multiple sclerosis. *Statistically significant differences in group means at $p<0.05$.

might play an important role in augmenting the asymmetry observed in this group of individuals with MS.

It is also important to note that our study participants were considered to have relatively low aerobic fitness based on oxygen uptake normative values [13]. The MS participants on average were considered to have "very poor" aerobic fitness and the control participants were of "fair" fitness levels [13]. This study suggests that during double-leg exercise testing, the stronger leg contributes a greater portion of overall work performed. Despite not

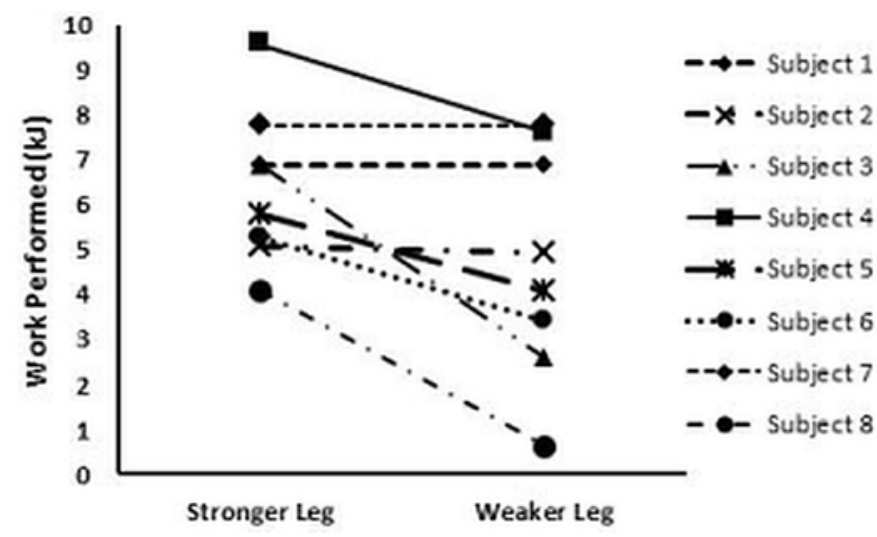

Figure 3.

Work performed during single-leg 5 min ride for stronger and weaker legs for 8 participants with multiple sclerosis.

reaching statistical significance between groups, the CI for the $\mathrm{VO}_{2}$ peak $(-14.3,0.7)$, along with the large effect size $(-0.98)$, gives the possibility that the mean $\mathrm{VO}_{2}$ peak could be 14.3 points lower in those with MS. Similarly, when comparing the nonsignificant group differences for peak workload (watts), the CI $(-86.6,5.2)$, along with the large effect size $(-0.97)$, gives the possibility that the mean peak workload could be 86.6 lower in those with MS. This observation, along with the significant submaximal performance differences between limbs in the individuals with MS, has potentially important implications when designing exercise programs and when evaluating program outcomes in people with MS. Exercise programs designed using traditional exercise testing and prescription guidelines might result in inappropriate exercise programming for individuals with lower-limb differences in strength and power. Our data also provide a possible explanation, in part, for why exercise responses may vary considerably across people with MS. When evaluating absolute work, our preliminary findings suggest possible mitochondrial function disparities [14] and even muscle recruitment patterns and a need for individualized testing and programs.

Assessment of individual limb function may reveal performance disparities that may otherwise go unrecognized. Testing for limb asymmetries might contribute to early implementation of therapies to attenuate potential deleterious effects on movement efficiency and daily activity performance. Disparities in lower-limb performance may contribute to heightened fatigue and reduced ability to meet fitness goals. Programs focused on improving 
Table 4.

Leg characteristics and performance: Composition (lean and fat mass), strength, and work performed during submaximal exercise for subjects with multiple sclerosis (MS) and controls.

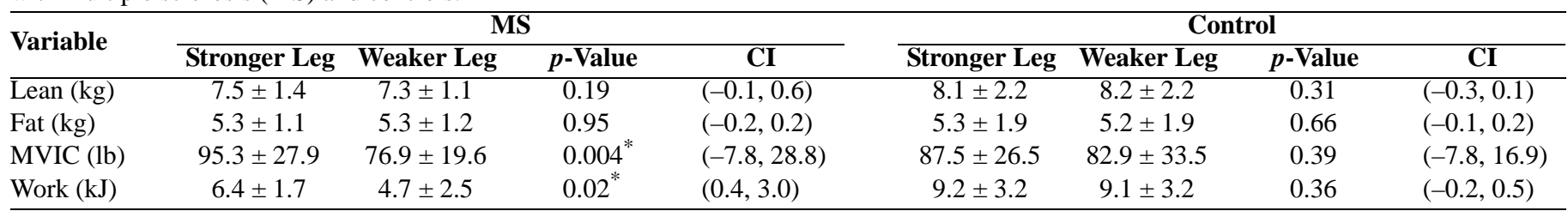

Note: Data are mean \pm standard deviation. Lean and fat mass from dual-energy X-ray absorptiometry. Work $=$ total work performed during 5 min submaximal single-leg cycling. Confidence interval (CI) represents differences between legs.

${ }^{*}$ Statistically significant differences between legs at $p<0.05$.

$\underline{\text { MVIC = maximal voluntary isometric contraction. }}$

unilateral performance decrements may enhance exercise training responses and contribute to overall health outcomes.

Our study is one of the first to quantify lower-limb bilateral physiological and functional differences during submaximal fixed workload and incremental exercises in individuals with MS. Considering the limited published literature, our small sample size and lack of metabolic measurements warrant further research to fully understand these differences. Additionally, this study involved ambulatory individuals with RRMS; therefore, larger studies with various levels of disease severity would be of interest to further quantify asymmetry and determine whether the magnitude of the differences in performance between legs changes or can be altered in individuals with MS. It is important to note that based on our sample of people with mild MS, there still was a wide variability in asymmetry. For example, some individuals had legs that were relatively similar in strength and function, whereas others had severe asymmetry that was not related to leg dominance (left vs right). Future studies might attempt to uncover the mechanism of this variability in the MS population.

\section{CONCLUSIONS}

In summary, total work during submaximal cycling was significantly different between legs in the MS group. These results highlight a need for individualized exercise testing when prescribing training programs. Because leg strength and function are fundamental to the maintenance of mobility and independence, additional research to fully understand causes and consequences of limb functional disparities on health, fitness, and function are warranted.

\section{ACKNOWLEDGMENTS}

\section{Author Contributions:}

Study concept and design: R. D. Larson, L. J. White.

Acquisition of data: R. D. Larson, D. J. Larson, W. M. Pryor. Analysis and interpretation of data: R. D. Larson, K. K. McCully, L. J. White.

Drafting of manuscript: R. D. Larson.

Critical revision of manuscript for important intellectual content: R. D. Larson, D. J. Larson, W. M. Pryor, K. K. McCully, L. J. White. Statistical analysis: R. D. Larson, D. J. Larson.

Administrative, technical, or material support: L. J. White,

K. K. McCully.

Study supervision: L. J. White.

Financial Disclosures: The authors have declared that no competing interests exist.

Funding/Support: This material was unfunded at the time of manuscript preparation.

Additional Contributions: We would like to thank all the participants for their involvement and cooperation during this study. Drs. Rebecca D. Larson and Daniel J. Larson are now affiliated with the Department of Health and Exercise Science, University of Oklahoma, Norman, Oklahoma.

Institutional Review: The University of Georgia Institutional Review Board approved this study.

Participant Follow-Up: The authors do not plan to inform participants of the publication of this study.

\section{REFERENCES}

1. Tantucci C, Massucci M, Piperno R, Grassi V, Sorbini CA. Energy cost of exercise in multiple sclerosis patients with low degree of disability. Mult Scler. 1996;2(3):161-67. [PMID:9345381]

2. Olgiati R, Jacquet J, Di Prampero PE. Energy cost of walking and exertional dyspnea in multiple sclerosis. Am Rev Respir Dis. 1986;134(5):1005-10. [PMID:3777662]

3. Chung LH, Remelius JG, Van Emmerik RE, Kent-Braun JA. Leg power asymmetry and postural control in women 
with multiple sclerosis. Med Sci Sports Exerc. 2008;40(10): 1717-24. [PMID:18799980] http://dx.doi.org/10.1249/MSS.0b013e31817e32a3

4. White LJ, Dressendorfer RH. Factors limiting maximal oxygen uptake in exertional monoparesis. Mult Scler. 2005; 11(2):240-41. [PMID:15794400] http://dx.doi.org/10.1191/1352458505ms1151oa

5. Larson RD, McCully KK, Larson DJ, Pryor WM, White LJ. Bilateral differences in lower-limb performance in individuals with multiple sclerosis. J Rehabil Res Dev. 2013; 50(2):215-22. [PMID:23761002] http://dx.doi.org/10.1682/JRRD.2011.10.0189

6. Thoumie P, Lamotte D, Cantalloube S, Faucher M, Amarenco G. Motor determinants of gait in 100 ambulatory patients with multiple sclerosis. Mult Scler. 2005;11(4): 485-91. [PMID:16042234] http://dx.doi.org/10.1191/1352458505ms1176oa

7. Lambert CP, Archer RL, Evans WJ. Muscle strength and fatigue during isokinetic exercise in individuals with multiple sclerosis. Med Sci Sports Exerc. 2001;33(10):1613-19. [PMID:11581542] http://dx.doi.org/10.1097/00005768-200110000-00001

8. Larson RD, White LJ. Asymmetrical hip bone density in multiple sclerosis. IJMSC. 2011;13(1):43-47. [PMID:24453704] http://dx.doi.org/10.7224/1537-2073-13.1.43

9. Kurtzke JF. Rating neurologic impairment in multiple sclerosis: An expanded disability status scale (EDSS). Neurology. 1983;33(11):1444-52. [PMID:6685237] http://dx.doi.org/10.1212/WNL.33.11.1444

10. Cohen JA, Cutter GR, Fischer JS, Goodman AD, Heidenreich FR, Jak AJ, Kniker JE, Kooijmans MF, Lull JM, Sandrock AW, Simon JH, Simonian NA, Whitaker JN. Use of the multiple sclerosis functional composite as an outcome measure in a phase 3 clinical trial. Arch Neurol. 2001; 58(6):961-67. [PMID:11405811]

http://dx.doi.org/10.1001/archneur.58.6.961

11. DeMyer WE. Technique of the neurological examination. 5th ed. New York (NY): McGraw Hill; 2004.

12. Hart S, Gabbard C. Brief communication: Bilateral footedness and task complexity. Int J Neurosci. 1996;88(1-2): 141-46. [PMID:9003971] http://dx.doi.org/10.3109/00207459608999819

13. Heyward VH. Advance fitness assessment and exercise prescription. 5th ed. Champaign (IL): Human Kinetics Publisher; 1998.

14. Brizendine JT, Ryan TE, Larson RD, McCully KK. Skeletal muscle metabolism in endurance athletes with nearinfrared spectroscopy. Med Sci Sports Exerc. 2013;45(5): 869-75. [PMID:23247709] http://dx.doi.org/10.1249/MSS.0b013e31827e0eb6

Submitted for publication September 3, 2013. Accepted in revised form July 8, 2014.

This article and any supplementary material should be cited as follows:

Larson RD, McCully KK, Larson DJ, Pryor WM, White LJ. Lower-limb performance disparities: Implications for exercise prescription in multiple sclerosis. J Rehabil Res Dev. 2014;51(10):1537-44.

http://dx.doi.org/10.1682/JRRD.2013.09.0191

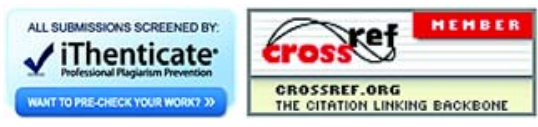


